\title{
Вопросы радикальности хирургического лечения злокачественных опухолей сердца
}

\author{
Р. М. Витовский ${ }^{1}$, Д. Н. Дядюн ${ }^{1}$, В. В. Исаенко ${ }^{1}$, В. Ф. Онищенко ${ }^{2}$,
} А. А. Пищурин ${ }^{2}$, В. В. Мартыщенко ${ }^{1}$

${ }^{1}$ Национальный институт сердечно-сосудистой хирургии имени Н. М. Амосова НАМН Украины, г. Киев, ${ }^{2}$ Национальная медицинская академия последипломного образования имени П. Л. Шупика, г. Киев

\section{The issues of the surgical treatment radicalism in surgical treatment of the heart malignancies}

\author{
R. M. Vitovsky ${ }^{1}$, D. N. Dyadyun ${ }^{1}$, V. V. Isaienko1, V. F. Onishchenko², \\ A. A. Pishchurin ${ }^{2}$ I. V. Martyshchenko ${ }^{1}$
}

${ }^{1}$ Amosov National Institute of Cardio-Vascular Surgery, Kiev,

${ }^{2}$ Shupyk National Medical Academy of Postgraduate Education, Kyiv

\section{Реферат}

Цель. Определить методы диагностики и хирургического лечения, направленные на максимально радикальное удаление злокачественной опухоли сердца.

Материалы и методы. В Институте сердечно-сосудистой хирургии имени Н. М. Амосова НАМН Украины с 1970 по 01.01.2018 г. проведено лечение 912 пациентам с опухолями сердца, в том числе 63 (6,9\%) пациентам со злокачественными новообразованиями сердца различного вида и локализации.

Результаты. Операции выполнены 62 больным, из них у 51 (82,3\%) - с использованием искусственного кровообращения. Госпитальная летальность при хирургическом лечении злокачественных опухолей сердца составила 22,6\% (умерли 14 больных). У 20 (32,3\%) больных, которым выполнены условно радикальные кардиохирургические вмешательства с использованием искусственного кровообращения, отмечен хороший гемодинамический эффект.

Выводы. Для достижения условной радикальности операции и максимального гемодинамического эффекта в целом ряде наблюдений необходимо применение расширенных методик реконструкции поврежденных структур сердца в сочетании с активной дезинтоксикационной терапией и безотлогательной химиотерапией.

Ключевые слова: злокачественные опухоли сердца; хирургическое лечение; радикальность.

\section{Abstract}

Objective. To determine the methods of diagnosis and surgical treatment, directed on maximally radical extirpation of the heart malignancy.

Materials and methods. The treatment of 912 patients, suffering tumors of the heart, was conducted in National Institute of Cardio-Vascular Surgery named after N. M. Amosov NAMS of Ukraine in 1970 - 01.01.2018 yr, including 63 (6.9\%) patients, suffering the heart malignancies of various kinds and localizations.

Results. The operations were performed in 62 patients, of them in $51(82.3 \%)$ - using artificial blood circulation. Hospital lethality in surgical treatment of the heart malignancies have constituted 22.6\% (14 patients died). In 20 (32.3\%) patients, in whom a conditionally radical cardio-surgical interventions, using artificial blood circulation, was done a good hemodynamic effect was noted. Conclusion. For achievement of a conditional radicalism of the operation and maximal hemodynamical effect in some observations the application of the extended procedures for the heart structures damaged in combination with active desintoxication therapy and urgent chemotherapy are mandatory.

Keywords: malignant tumors of the heart; surgical treatment; radicalism.

Проблема хирургического лечения злокачественных опухолей сердца (3ОС) остается актуальной и вызывает особый интерес у кардиохирургов. Впервые удачное кардиохирургическое лечение фибросаркомы правого предсердия (ПП) описали L. A. Longino и I. A. Meeker в 1953 г. [1]. В мировой литературе приведены наблюдения успешного лечения этой тяжелой патологии [2 - 4]. По мере развития и широкого использования в послеоперационном периоде радио- и химиотерапии отдаленные результаты операций можно признать относительно удовлетворительными, хотя успех лечения пропорционально зависит от стадии процесса и чаще достигается на ранних этапах заболевания [3-5].
Несмотря на то, что хирургия 3ОС не является весьма распространенным разделом хирургии, она остается малоизученной ввиду небольшого числа наблюдений и успешных кардиохирургических вмешательств.

Раннюю диагностику 3ОС у 50 - 80\% пациентов затрудняет асимптоматичность течения заболевания, и на момент диагностики этой патологии выявляют множественные регионарные или отдаленные метастазы, что в свою очередь ухудшает как общее состояние пациентов, так и прогноз последующего хирургического лечения [6, 7].

Госпитальная летальность в данной группе пациентов крайне высока (20 - 50\%). Чаще всего это обусловлено распространенностью поражения сердца злокачествен- 
ным процессом на момент установления диагноза, что в свою очередь приводит к необходимости расширения объемов хирургического лечения, включая протезирование или реконструктивные вмешательства на клапанах сердца, пластическую коррекцию сердечных структур, коронарное шунтирование и имплантацию кардиостимулятора при необходимости [7 - 9].

В настоящее время многие кардиохирурги ведущих клиник мира видят выход из создавшегося столь серьезного положения в ранней дифференциальной диагностике злокачественного опухолевого роста, а также в безотлагательной пересадке сердца, что позволяет добиться значительно лучших отдаленных результатов [7 - 9]. Однако, поскольку с пересадкой сердца связаны сложные для различных стран вопросы законодательного и морально-этического характера, остается актуальной проблема радикальности удаления 3ОС.

Цель иссследования: поделиться многолетним опытом хирургического лечения 3ОС в контексте обеспечения максимальной радикальности операции и связанных с этим дополнительных хирургических манипуляций.

\section{Материалы и методы исследования}

В Институте сердечно-сосудистой хирургии имени $\mathrm{H}$. М. Амосова НАМН Украины с 1970 по 2018 г. прооперированы 912 пациентов с опухолями сердца, из них у 63 (6,9\%) пациентов были злокачественные новообразования сердца различного вида и локализации.

Женщин было 34 (54\%), мужчин - 29 (46\%). Возраст пациентов колебался от 12 дней до 71 года и в среднем составил $(34,5 \pm 8,6)$ года.

Всем больным было проведено полное клиническое обследование. Из инструментальных методов исследования использовали электрокардиографию, рентгенологическое исследование, эхокардиографию, коронаровентрикулографию (по показаниям), компьютерную (КТ) и магнитно-резонансную (МРТ) томографию (при необходимости). Приоритетным методом для установления диагноза была комплексная эхокардиография, дополненная данными КТ. Всем больным в послеоперационном периоде проводили морфологическое исследование опухолей, что дало возможность с точностью определить их злокачественность.

Наиболее часто выявляли ангиосаркомы (у 20 больных), рабдомиосаркомы (у 9) и злокачественные миксомы (у 9), несколько реже - лейомиосаркомы (у 7). Фибро- саркомы наблюдали у 6 больных, ангиофибросаркомы у 3, хондросаркомы - у 2, злокачественные гистиоцитомы - у 2, липосаркому - у 1, лимфому - у 1 больного. У 2 больных было выявлено и подтверждено гистологически метастатическое поражение сердца.

Наиболее часто опухоли локализовались в левом предсердии (ЛП) - у 27 (42,9\%) больных. На втором месте по частоте изолированного поражения злокачественными новообразованиями был правый желудочек (ПЖ) - у 12 (19\%) больных. Изолированное поражение левого желудочка (ЛЖ) и ПП наблюдали соответственно у 5 (7,9\%) и 8 (12,7\%) больных. Множественное поражение, когда опухоль локализовалась в двух и более камерах сердца, отмечено у 11 (17,5\%) пациентов. Таким образом, по нашим наблюдениям, левые отделы сердца были поражены злокачественными новообразованиями в полтора раза чаще, чем правые, - соответственно у 32 (50,8\%) и 20 (31,7\%) пациентов.

Хирургическое лечение проведено 62 пациентам с 3ОС. Умерли 14 (22,6\%) больных. В течение первых суток пребывания в клинике умер 1 пациент, у него диагностирована рабдомиосаркома ЛП и Лж.

\section{Результаты}

Из 62 операций 51 (82,3\%) выполнена с использованием искусственного кровообращения в условиях гипотермии и применения холодовой фармакологической кардиоплегии. У 1 (1,6\%) пациента опухоль ЛЖ удалось частично удалить на работающем сердце. Эксплоративную торакотомию с последующим забором материала для биопсии выполнили 10 (16,1\%) больным. У этих пациентов было выявлено значительное поражение опухолевым процессом с распространением на близлежащие ткани и органы (метастазы), что делало последующее хирургическое лечение невозможным, и они вынужденно были признаны неоперабельными.

Доступ и объем хирургического вмешательства по поводу 3ОС определяли сугубо индивидуально, руководствуясь такими критериями: локализация, распространенность, миокардиальная инвазия, степень вовлечения клапанного аппарата сердца.

Главной целью хирургического лечения первичных 3ОС являлось восстановление гемодинамики и достижение условной радикальности операции путем максимального удаления неопластической ткани, что удавалось далеко не всегда (см. маблииу).

\begin{tabular}{|c|c|c|c|c|c|c|c|c|c|c|c|c|c|}
\hline \multicolumn{14}{|c|}{ Радикальность операций (n=62) в зависимости от вида злокачественной опухоли } \\
\hline \multirow{2}{*}{$\begin{array}{c}\text { Варианты радикальности } \\
\text { операций }\end{array}$} & \multicolumn{11}{|c|}{ Вид опухоли } & \multicolumn{2}{|c|}{ Всего } \\
\hline & $A C$ & PMC & лМС & $3 \mathrm{M}$ & $\Phi C$ & XC & AФC & $3 \Gamma$ & л & лС & МПС & абс. & $\%$ \\
\hline Условная радикальность & 4 & 3 & 2 & 5 & 3 & 1 & 2 & - & - & - & - & 20 & 32,3 \\
\hline Частичное удаление & 11 & 4 & 5 & 3 & 1 & 1 & 1 & 2 & 1 & 1 & 2 & 32 & 51,6 \\
\hline Эксплоративная торакотомия & 5 & 2 & - & 1 & 2 & - & - & - & - & - & - & 10 & 16,1 \\
\hline Итого ... & 20 & 9 & 7 & 9 & 6 & 2 & 3 & 2 & 1 & 1 & 2 & 62 & 100 \\
\hline 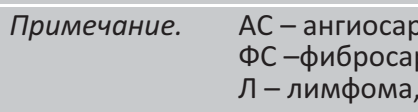 & & & & & & & & & & & & & \\
\hline
\end{tabular}


Косвенно судить о сложности оперативного вмешательства и определить его последующий характер возможно по данным эхокардиографии или КТ, которые позволяют установить распространенность опухолевого процесса на дооперационном этапе. Однако окончательное решение об объеме вмешательства принимали непосредственно при визуальном контроле в момент его выполнения. Условием достижения условной радикальности операции являлось максимальное удаление новообразования до видимо здоровых тканей. Говорить об абсолютной радикальности выполнения операции, на наш взгляд, не представляется возможным ни в одном наблюдении, что связано с неочевидным распространением злокачественного процесса. Из приведенных в таблице данных видно, что меньше чем у половины больных с 3ОС (32,3\%) при удалении новообразования удавалось иссечь его до видимых здоровых тканей, не пораженных опухолью.

По объему оперативного вмешательства возможно получить представление о степени миокардиальной инвазии разными злокачественными опухолями. При ангиосаркомах и рабдомиосаркомах примерно в равном количестве выполнили радикальные и паллиативные операции, эксплоративную торакотомию - соответственно у 5 и 2 больных. Эти данные свидетельствуют о склонности указанного вида опухолей к быстрому инвазивному росту. Из 8 пациентов со злокачественными миксомами, радикальность оперативного вмешательства была соблюдена у 5 . При лейомиосаркомах у 5 из 7 пациентов выполнены паллиативные вмешательства, направленные на устранение обструкции кровотока путем частичного иссечения злокачественного образования сердца, и лишь у 2 пациентов удалось радикально удалить образование. Это свидетельствует о быстром и массивном поражении миокарда данным видом опухоли и невозможности соблюдения радикальности вмешательства у большинства таких больных.

Часть оперативных вмешательств по поводу удаления первичных 3ОС выполняли с дополнительными хирургическими манипуляциями, которые требовались ввиду массивного распространения неопроцесса на различные структуры сердца. Пластику стенок предсердий выполнили в 5 наблюдениях, пластику межпредсердной перегородки - в 6, восстановление целостности задней стенки верхней полой вены - в 1, пластические операции на клапанах сердца - в 8, протезирование клапанов - в 6 (в 3 - митрального и в 3 - трикуспидального).

Необходимость выполнения таких дополнительных манипуляций во время удаления 3ОС возникала значительно чаще в сравнении с оперативными вмешательствами по поводу доброкачественных опухолей сердца. Дополнительные манипуляции обусловлены стремлением хирурга повысить радикальность хирургического вмешательства.

Необходимость расширить объем операции возникла у 22 (43,1\%) больных, оперированных с использованием искусственного кровообращения.

Кроме того, у 14 (22,6\%) пациентов злокачественный опухолевый процесс распространялся на клапанный аппарат сердца, что привело к необратимым деструктивным изменениям и дисфункции сердечных клапанов. С целью достижения радикальности оперативного вмешательства и улучшения гемодинамического эффекта потребовалась частичная или полная резекция пораженных клапанных и подклапанных структур с выполнением различных пластических операций (у 8 больных) или протезирования клапана (у 6).

Например, пластическое вмешательство на митральном клапане было выполнено пациенту Т. (и.б. № 321 , 2008 г.) с рабдомиосаркомой ЛЖ, которая поражала заднюю створку, хорды и папиллярные мышцы. Помимо удаления самой опухоли, была выполнена секторальная резекция задней створки с частью хорд и папиллярных мышц митрального клапана с последующей пликацией клапанного кольца, что позволило добиться компетентности митрального клапана. У пациента К. (и.б. № 321, 2017 г.) с метастатическим поражением сердца во время ревизии было выявлено полное вовлечение в процесс клапанного аппарата и подклапанных структур трехстворчатого клапана, что потребовало, помимо удаления самой опухоли, иссечения пораженного клапана с последующим его протезированием.

Сравнительный анализ данных показал несущественное влияние дополнительных манипуляций, включая реконструктивные процедуры на различных сердечных структурах, на риск самого оперативного вмешательства. Из 22 больных, оперированных по расширенным методикам, умерли 5 (22,7\%), а из 29 больных, оперированих без применения дополнительных манипуляций, - 9(31\%).

\section{Обсуждение}

Причиной смерти практически всех умерших больных была обширность патологического процесса с тяжелым интоксикационным синдромом, что и обусловливало тяжесть их состояния.

Исходя из изложенного, для достижения максимального эффекта хирургического лечения 3ОС необходимо выполнять наиболее полное радикальное иссечение опухолевой ткани, применяя при необходимости расширенные методики с целью реконструкции поврежденных структур сердца и улучшения таким образом гемодинамического эффекта. При этом в ближайшем послеоперационном периоде немаловажное значение имеет применение активной дезинтоксикационной терапии и безотлогательной химиотерапии в дальнейшем.

Первичные 3ОС - достаточно разнообразная онкологическая патология, что подтверждает анализ накопленного материала. Полиморфизм их локализации и течения значительно усложняет процесс диагностики, что в большинстве наблюдений приводит к запоздалому выявлению и поступлению больных в кардиохирургические центры для хирургического лечения. Операции при данном виде патологии выполнять технически сложно, объем их определяется сугубо индивидуально в зависимости от локализации, степени миокардиальной и клапанной инвазии и наличия метастатических поражений.

\section{Выводы}

Из представленных данных следует, что у больных с СО3 далеко не всегда удается добиться радикальности 
операции. Несмотря на это, главная цель хирургического лечения 3ОС - максимальное удаление опухолевых тканей с целью достижения условной радикальности операции и выполнение при необходимости дополнительных реконструктивных процедур на поврежденных структурах сердца для обеспечения лучшего гемодинамического эффекта.

\section{Підтвердження \\ фінансування}

Ініціативно-пошукова робора. Фінансування публікації - приватна особа (В. В. Ісаєнко).

\section{Внесок авторів}

Р. М. Вітовський - аналіз отриманих даних, написання тексту; Д. Н. Дядюн - збирання та опрацювання матеріалу; В. В. Ісаєнко - аналіз отриманих даних, написання тексту; О. А. Піщурін - концепсія і дизайн дослідження; В. Ф. Онищенко - концепсія і дизайн дослідження; I. В. Мартищенко - збирання та опрацювання матеріалу.

Всі автори прочитали та схвалили остаточний варіант рукопису.

\section{Конфлікт інтересів}

Автори декларують відсутність конфлікту інтересів.

Згода на публікацію

Всі автори дали згоду на публікацію цього рукопису.

\section{References}

1. Longino LA, Meeker IA Jr. Primarycardiac tumors in infancy. J Pediatr. 1953 Dec;43(6):724-31. PMID: 13109673

2. Knyshov GV, Vitovskij RM, Zaharova VP. Opuholi serdca. Kyev: Presa Ukrainy; 2005. 256 p. [In Russian].

3. Yin L, He D, Shen H, Ling X, Li W, Xue Q, Wang Z. Surgical treatment of cardiac tumors: a 5-yearexperience from a singlecardiac center. J Thorac Dis. 2016 May;8(5):911-9.doi: 10.21037/jtd.2016.03.87.

4. Oliveira GH1, A-Kindi SG, Hoimes C, Park SJ. Characteristics and Survival of Malignant Cardiac Tumors: A 40-Year Analysis of $>500 \mathrm{~Pa}-$ tients. Circulation. 2015 Dec 22;132(25):2395-402. doi: 10.1161/CIRCULATIONAHA.115.016418.

5. Burke A, Tavora F.The 2015 WHO Classification of Tumors of the Heart and Pericardium.J Thorac Oncol. 2016 Apr;11(4):441-52. doi:10.1016/j. jtho.2015.11.009.

6. Hudzik B, Miszalski-Jamka K, Glowacki J, Lekston A, Gierlotka M, Zembala M, et al. Malignant tumors of the heart. Cancer Epidemiol. 2015 Oct;39(5):665-72. doi: 10.1016/j.canep.2015.07.007.

7. Mkalaluh S, Szczechowicz M, Torabi S, Schmack B, Sabashnikov A, Dib B, et al. Surgical Treatment of Cardiac Tumors: Insights from an 18Year Single-Center Analysis. Med Sci Monit. 2017 Dec 31;23:62016209. PMID: 29289957.

8. Lestuzzi C, De Paoli A, Baresic T, Miolo G, Buonadonna A. Malignant cardiac tumors: diagnosis and treatment. Future Cardiol. 2015 Jul;11(4):485-500. doi: 10.2217/fca.15.10.

9. Yanagawa B, Mazine A, Chan EY, Barker CM, Gritti M, Reul RM, et al. Surgery for Tumors of the Heart. Semin Thorac Cardiovasc Surg. 2018 Sep 8. pii: S1043-0679(18)30177-1. doi: 10.1053/j. semtcvs.2018.09.001. 